\title{
Zur pathologischen Anatomie der Chorioidea
}

\author{
von \\ Dr. C. Schweigger.
}

Indem ich in Bezug auf die eitrige Choroiditis auf meine früheren Darstellungen (A. f. O. B. V. Abth. 2 pag. 210) verweise, will ich hier nur noch binzufügen, dass nicht selten gleichzeitig mit der Eiterbildung sehr beträchtliche Errgüsse gerinnungsfähiger Flüssigkeiten im Choroidalstroma angetroffen werden, wodurch das letztere zu einer beträchtlichen Dicke aufgetrieben werden kann; die angeschwollene Choroidea ragt dann mit einer unebenen, hügelichen Oberfläche in das Innere des Bulbus hinein. Diese Ergüsse können im Choroidalstroma in nicht unbeträchtlicher Menge vorhanden sein und eine sehr deutliche Dickenzunahme desselben bedingen, ohne dass Netzhautablösung eintritt; bei weitem in den meisten Fällen tritt allerdings Netzhautablösung ein.

Das Schicksal der abgelösten Netzhautist grösstentheils von der Gestalt ablängig, welche sie im abgelösten 7ustande einnimmt. Wird sie in ihren hinteren Partieen strangförmig comprimirt, während die vorderen nach der tellerförmigen Grube hin zusammengedrängten Massen eine unebene, 
hügeliche Oberfläche zeigen, so leidet ihre Structur bedeutend mehr, als wenn sie glatt zusammengelegt wird oder eine wenigstens annähernd regelmässige Gestalt behält.

Aber auch ohne abgelöst zu sein, kann die Netzhaut, bei Choroiditis, wahrscheinlich durch eine Art von Frweichung, functionsunfähig werden.

Endlich ist es mir wahrscheinlich geworden, dass durch eitrige Choroiditis eine Atrophie der Chorio. capillaris und dadurch Atrophie des Bulbus eingeleitet werden kanı.

Unter dem Namen der ect at is chen Processe fassen wir alle diejenigen Vorgänge zusammen, welche auf einer Yermehrung des Glaskörper-Volumens beruhen. Ob der den hierher gehörigen Krankheitsgruppen zu Grunde liegende Process der Glaskörpervermehrung als ein entzündlicher Vorgang bezeichnet werden darf, ist zweifelhaft, und jedenfalls ist in vielen Fällen die Anschauung durchaus berechtigt, dass entzündliche Processe, welche an solchen Augen auftreten, eben die Folge der Volumsvermchrung des Glaskörpers sind. Das wenigstens kam man gewiss behaupten, dass bei weitem der grösste Theil der anatomischen Veränderungen, welche man bei ectutischen Processen findet, nicht Lrsache, sondern Folge der Ectasie sind. Diese Veränderungen zeigen vorwiegend einen atrophischen Charakter und sind wahrscheinlich direct abhängig von der Dehnung, welcher die Choroidea ausgesetzt ist. Der Gang dieser Atrophie pflegt der Art zu sein, dass zunächst die verästelten Pigmentzelleul der Choroidalstroma sich entfärben und nach und nach verschwinden. Bald fängt auch dic cho riocapillaris an $z u$ atrophiren, während gleichzeitig das Pigmentepithel theils sein Pigment verliert, theils in verschiedentlicher Weise unregelmässig wird. Auch die griisseren Gefässe obliteriren und schliesslich bleibt von der 
Choroidea nichts übrig als die feinsten Fäserchen, welche als sog. elastisches Netzwerk einen Theil des Choroidalstroma ausmachen, die Glaslamelle und ein unvollständiger und unregelmässiger Pigmentüberzug; auch diese Gewebsreste können endlich noch verschwinden, so dass nichts weiter als eine dünne, structurlose durchsichtige Membran zurückbleibt. Gewöhnlich, aber nicht immer, ist die in dieser Weise atrophirte Choroidea fest mit der Sclera, und da, wo eine starke locale Ectasic der Bulbuswandungen, ein sogenanntes Scleral - Staphylon, vorhanden ist, auch noch mit der Netzhaut verwachsen. An den so veränderten Stellen ist nun auch dic Sclera mit verdünnt, oder vielmehr umgekehrt, an den Stellen, wo die Sclera nachgiebiger ist und desshalb ausgedehnt und verdünnt wird, erleidet auch die Choroidea analoge Veränderungen.

Es ist bekannt, dass bei allgemeiner, wenn aueh nicht in allen Duchmessern gleicher Ausdehnung des Bulbus durch Volumsvermehrung des Glaskörpers die consecutive Atrophie der Choroidea gerade an einer bostimmten Stelle, dem äusseren Rande der Papille, am frühesten einzutreten pflegt.

Da wo es mir gelang, die atrophische Choroidea an der Sehnerven-Peripherie in einiger Ausdehnung unversehrt von der Sclera abzulösen, fand sich die in Rede stehende Choroidalpartie in ein sehr dünnes, streifiges, durchsichtiges, absolut gefässloses Gewebc verwandelt, während die angrenzende Choroidea einen geringen Grad von Atrophie zeigte, der weiter nach dem Aequator hin allmählig einem normalen Verhalten Plat machte. Ein entzündlicher Character dieser Veränderungen liess sich in keiner Weise constatiren, es handelte sich lediglich um eine Atrophie, als deren Ursache wir die durch die Vergrösserung des Glaskörpervolumens bedingte Iehnung der Choroidea ansehen müssen. Bei- 
läufig will ich hier bemerken, dass ich genau dieselbe Form von Atrophie der Choroidea, nur in bedeutend geringerer Ausdehnung, auch neben Excavationen der Papille vorgefunden habe, so dass der schmale, helle Ring, den man manchmal die excavirte Papille umgeben sieht, wohl auch auf eine umscltriebene Atrophie der Choroidea zu beziehen sein dürfte. Dass die bei Myopie durch Sehachsen-Verlängerung so gewöhnliche sogenannte „Sclerotico Choroiditis posterior" überhaupt ein entzündliches Leiden sei, ist meiner Ceberzeugung nach durch anatomische Untersuchungen noch keineswegs bewiesen, die ophthalmostiopisch sichtbaren Veränderungen sind jedenfalls lediglich atrophischer Natur, und am allerwenigsten kann etwa davon die Rede sein, die in der Limgebung des Sehnerven vorhandenen Veränderungen als Ursache der Sehachsen-Verlängerung anzusehen. Die Frage kann nur die sein, warum bei einer allgemeinen Dehnung der Bulbuswandungen durch Glaskörper-Vurmehrung gerade an dieser einen Stelle, so constant sich zuerst die Folge dieser Dehnung, die Atrophie der Choroidea, entwickelt. Es lassen sich dafür meines Erachtels folgende Ursachen anführen: Man kann annehmen, dass eine Membran, welche in ihrer Totalität einer Dehnung unterliegt, davon abhängige Ernährungsstörungen da zuerst erleiden wird, wo sie am wenigsten im Stande ist, durch Verschiebung sich der Dehnung zu entziehen. Die vorderen Abschnitte der Choroidea sind nun mit der Sclera nur in lockerer Verbindung, sie können sich daher der Dehnung einigermassen entziehen indem sie (sammt der Iris) etwas nach hinten rücken - dies könnte erklären, warum man bei Myopen nicht selten eine tiefere vordere Kammer und etwas weitere Pupillen antriffit - an der Eintrittsstelle des Sehnerven dagegen ist die Choroidea fest, heinahe untrennbar mit der Sclera verwachsen und schiebt sogar einzelne pigmentlose Fäserchen in das Ge- 
webe der lamina cribrosa hinein; hier kaun sich die Choroidea also nicht durch Verschiebung der Dehnung entziehen; wenn nun ferner, wie es meistens zu geschehen scheint, der Längsdurchmesser des Bulbus in diesen Fällen die relativ stärkste Ausdehnung erleidet, so würde dies auch erklären, warum dic consecutive Atrophie der Choroidea meistens gerade an der äusseren Seite der Papille am häufigsten auftritt.

Ein anderes mechanisches Moment scheint mir in den Bewegungen des Bulbus zu liegen. Der Bulbus findet bei seinen Drehungen innerhalb der 'Tenon'schen Kapsel nirgends einen erhehlichen Widerstand, nur an der Eintrittsstelle des Sehnerven finden Verhältnisse statt, welche Erwähnung verdienen. Der Bulbus muss bei allen seinen Bewegungen den Sehnerven mitnehmen, was nicht denkbar ist ohne einen gewissen, wenn auch noch so geringen mechanischen Widerstand. Da der Sehnerv sich nach innen vom hinteren Pol inserirt, so muss dieser Widerstand um so grösser werden, jo mehr sich der hintere Pol im Dienste des Sehactes nach aussen bewegt, d. h. je grösser die beanspruchte Convergenz der Augenachsen ist. Dieses mechanische Moment, welches für normale Augen gewiss äusscrst unbedeutend ist, kann doch in's Gewicht fallen für myopische Augen, deren Selera schon verdünnt und desshalb dehnbarer ist, und welche z. B. beim Arbeiten eine stärkere Convergenz anbaltend beanspruchen müssen. Aus diesen Gründen dürfte es zweckmässig sein, solchen Nyopen durch prismatische Brillen einen Theil der zum Arbeiten nothwendigen Convergenz abzunehmen.

Fin eigentliches Staphyloma posticum, d. h. eine von den übrigen Bulbuswandungen mehr oder weniger scharf abgegrenzte locale Fctasie scheint nur selten zu Stande zu kommen, und man sollte mit dem Namen des Staphyloma posticum weniger freigebig sein. Bei weitem 
in den meisten Fällen von Myopie mit Atrophia Choroideae circa papillam kaun man sich ophthalmoskopisch überzeugen, dass ein eigentliches Staphyloin, d. h. eine mehr odler weniger scharf begrenzte locale Ectasie der Bulbuswandungen nicht stattfindet; die ophthalmoskopischen Kennzeichen, aus denen wir Niveaudifferenzen im Augeuhintergrunde überhaupt beurtheilen, sind natürlich auch hier entscheidend, und seitdem ich angefungen habe, in allen Fällen von Myopic genau hierauf zu achten, glaube ich gefunden zu haben, dass in den Fällen, wo ausuahmsweise wirklich eine Staphyloma posticum, d. h. eine locale Ausbuchtung der Bulbuswandungen neben dem Sehnerven vorhanden ist, auch die Sehschärfe erheblicher herabgesetzt zu scin pflegt als sonst bei gleichen Graden von Myopie.

Bilden sich bei allgemeiner Volumszunahme des Glaskörpers locale Fctasien, so sind dafür eben auch locale Gründe aufzusuchen. So liegt es z. B. auf der Hand, warum Ectasien, welche sich in der Gegend des Aequator bulbi entwickeln, am leichtesten zwischen den musculi recti sich ausbilden. Dass auch an der Eintrittsstelle des Sehnerven eine locale Praedisposition besteht, ist in dem eben Besprochenen enthalten. Für die seltener vorkommenden sog. Intercalar-Ectasien, welche sich zwischen den Hornhautrand und die Firsten der Ciliarfortsätze einschieben, indem sie letztere nach hinten drängen, fand ich in einem Falle als wahrscheinliche 10cale Ursache der Letasie eine Irido-Dialyse. (Vergl. Arch. f. Ophth. B. VI 1 pag. 166). lis handelte sich dabei um einen Fall von bedeutender allgemeiner Ausdehnung der Bulbuswandungen; der Pupillarrand der Iris war fixirt durch Verwachsung mit der in die vordere Kammer luxirten Linse, der Ciliarrand, durch die Dehnung der Choroidea stark nach hinten gezogen, die Iris dailurch gleichfalls ausgedehnt und verdünnt; unter dem 
linflusse dieser Dehnnng hatte sich endlich am oberen Rande der Iris eine Dialyse gebildet, und die dadurch blossgelegte Stelle der Sclera erfuhr nun eine stärkere locale Ausdehnung, wodurch die Ciliarfortsätze nach hinsell gedrängt wurden.

Wir sind bisher yon der Vornussetzung ausgegangen, dass bei Volumszunahme des Glaskörpers die Sclera auch dehnbar genug sei, um dem vermehrten Bulbusinhalt hinreichend Raum za gewähren. Die Sclera spielt bei diesen Processen eine sehr wichtige Rolle, auf welche ich bereits bei einer früheren Gelegenheit hingewicsen habe. (Deutsche Klinik 1860 No. 25 pag. 242). Frmangelt nämlich die Sclera ihrer normalen Dehnbarkeit, ist sie z. B. durch senile Veränderungen oder durch innere dyscratische Ursachen, welche, wie z. B. Arthritis, die fibrösen Häute afficiren; weniger dehmbar geworden, und erfolgt nun doch eine Volumszunahme des Glaskörpers, so nuss, wenn die Scleri nicht nachgiebt, der intraoculare Drnck in erheblicher Weise steigen; endlich findet sich ein locus minoris resistentiae in der lamina cribrosa, die schon deshalb cine schwach befestigte Stelle der Sclera ist, weil hier das Scleralgewebe durch dic Nervenbündel des Opticus durchbohrt und auseinander gedrängt wird. Es entwickelt sich so die ganze Reihe der glaucomatösen Symptome und Excavation des Sehnerven. Dass auch Glaucom, sich selbst überlassen, schliesslich denn doch noch durch weitere Volumszunahme des Glaskörpers nicht selten zu Ectasien der Bulbuswandungen führt, ist bekannt genug und ein neuer Beweis für die Verwandtschaft des Glaucoms mit anderen ectatischen Processen.

Räthselhaft und von Haffmanns (Bijtrage tot de Kennis von det Glaucom -- siehe auch A. f. O. B. VIII Abth. 2 pag. 160) mit Recht hervorgehoben, ist in dieen Fällen das Verhalten des Glaskörpers; während sonst 
cin einigermassen anhaltender Fingerdruck genügt, um einen Theil des Glaskörpers zur Resorption zu bringen, während wir nicht selten ohne äusserlich sichtbare Störungen in wenigen Stunden grosse Netzhautablösungen entstehen sehen, die ja doch auch eine schnelle Resorption des Glaskörpers voraussetzen, sehen wir fjier den Glaskörper hartnäckig jeder Resorption widerstehen. Dennoch kann auch in glaucomatösen Augen Netzhautablösung zu Stande kommen: Ein Auge, welches durch wiederholte glaucomatöse Entzündungen erblindet war, wurde während cines neuen heftigen Entzündungsanfalles von Prof. v. Graefe exstirpirt; die Section ergab neben einer tiefen Druckexcavation des Sehnerven eine frische Netzhautablösung.

Bei Gelegenheit des Widerstandes, den der Glaskörper dem Zustandekommen von Netzhautablösungen eigentlich entgegensetzen sollte, wollen wir hier noch kurz die Netzhautablösung durch Glaskörperschrumpfung erwähnen, auf die H. M üller zuerst die Aufmerksamkeit gelenkt hat. Es dürften noch weitere Lntersuchungen nöthig sein, um festzustellen, in einer wie grossen Quote von Fällen Glaskörperschrumpfung als Lrsache von Netzhautablösung ophthalmoskopisch zu constatiren ist; dagegen habe ich einen Fall anatomisch zu untersuchen Gelegenheit gehabt, wo dieselbe ganz evideut war: An einem mit Linsenluxation behafteten Auge wurde von Professor v. Graefe eine Iridectomie verrichtet, während derselben erfolgte (eben wegen der Linsenluxation) etwas Glaskörpervorfall. Die Wunde heilte vollständig gut, aber etwa 8 Tage nach der Operation zeigte sich eine Sehstörung, welche, wie sich nachher ergab, auf Netzhautablösung beruhte. Einige Jahre später nöthigten unerträgliche subjective Lichterscheinungen zur exstirpatio bulbi. Das Auge wurde nach der Erhärtung im horizontalen Meridian durchschnitten, und nun zeigte es sich, wie in die am inneren Hornhautrande liegende Opera- 
tionsnarbe dichte streifige Glaskörpermassen hineingewachsen waren und von hier aus quer durch den Glaskörperraumn hindurchgehend, in einer ungefähr äquatorialen Stelle die gegenüberliegende äussere Netzhauthälfte erreichten, der sie stellenweise fest adhärirten; die mit ihnen verwachsene Retinalpartic war scharfwinklig nach vorn gezogen. Die Netzhautablösung war natürlich in grösserem Umfange vorhanden, aber keine totale, ein Theil der inneren Netzhauthälfte lag noch an. Der Glaskörper zeigte sich bei der mikroskopischen Untersuchung in den vorderen Partieen reich an zelligen File menten von zum Theil colossaler Grösse, welche stellenweise zu strangförmigen Massen zusammengefügt waren. Die Retina war in ihrer Structur verhältnissmässig wenig verändert, auch in der abgelösten Partie fanden sich noch einige Ganglienzellen, auf der Oberfläche der Choroidea einige schwarze Flecke durch Pigmentanhäufung in den Epithelien. Die genaueren Verhältnisse der Linsenluxation liessen sich (da die Untersuchung von vorn herein auf das eigenthïmliche Verhalten der Netzhautablösung gerichtet war) nicht hinreichend sicher zur Darstellung bringen, doch war eine Verlängerung dex Zonula mit Wahrscheinlichkeit anzunehmen. Da Glaskörpervorfall bei Cataract-Extractionen nicht selten vorkommt, ohne zur Netzhautablösung zn führen, so dürfte der hier wohl nicht zu bezweifelnde Zusammenhang zwischen dem Glaskörpervorfall und der Netzhantablösung doch wohl nur in einem praeexistirenden Glaskörperleiden seine Erklärung finden.

Von grosser Wichtigkeit für das ophthalmoskopische Bild des Augenhintergrundes ist das ChoroidalEpithel. Wie sehr diese einfache Zellenschicht das dahinter liegende Choroidalstroma verdeckt, davon kann man sich anatomisch sehr leicht überzeugen; man braucht nur die Choroidea ohne alle weitere Präparation son der Sclera abzuziehen und nun bei schwacher Ver- 
grösserung Stücke mit unverschrtem lipithel mit solchen zu vergleichen, bei denen man die Fpithelialschicht künstlich entfernt hat. Manchmal sieht man auch ophthalmoskopisch bei ausgedchnten Choroidalveränderungen einzelne Bezirke der Choroidea, in denen das ChoroidalEpithel entfärbt oder ganz verloren gegangen ist, und kaun dann die Deutlichkeit, mit der hier das sonst unveränderte Choroidalstroma mit seinen Gefässen u. s. w. erscheint, mit dem mehr weniger verschleierten Ausselen verglichen, welches es an den Stellen darbictet, an welchen das Epithel noch unverändert ist. Yon den physiologischen Verhältnissen des Choroidal-Epithels will ich hier nur erwähnen, class diese die ganze Lebensdaner hindurch persistirende Zellenschicht, welche bei Neugebornen (bei sehr geringer Pigmentirung des Choroidalstroma's) eine sehr dunkle Färbung zeigt, bei Frwachsenen dagegen durchschnittlich heller und im ganzen Augenhintergrunde gleichmässig gefärbt zu sein pflegt, in der Gegend der macula lutea durch eine dunklere Färbung des Pigment-Epithels sich auszeichnet. Nach Entfernung der Retina sieht man hier cinen der Ausdehnuıg der macula lutea entsprechenden dunkleren Fleck, der in einer stärkeren Pigmentfüllung des lipithels seinen Grund hat, und an der Peripherie ohne scharfe Grenzen in den individuellen Farbenton des Pig-ment-Epithels übergeht. Diese dunklere Färbung des Pigment-Epithels in der Gegend der macula lutea ist für die ophthalmoskopische Cntersuchung wohl zu beachten, da sie in manchen Fällen dieser Stelle eine vom übrigen Augenhintergrunde so verschiedene Färbung verleiht, dass diagnostische Irrthümer (Verwechselungen mit Haemorrhagien etc.) dadurch veranlasst werden können. Vorn in der Gegend der ora serrata ist das Pigmentepithel gewöhnlich weniger regelmässig als auf der iibrigen Choroidea angeordnet, während der Pigmentüberzug 
der Ciliarfortsätze sowohl durch die mehr rundliche Form seiner Zellen, als durch die dunkle Färbung les darin enthaltenen Pigmentes den Uebergang zu den Pigmentzellen des Uvealblattes der Iris vermittelt. Fest ver. wachsen mit dem schwarzen Epithel des Ciliarkörpers zeigt sich eine Schicht pigmentloser länglicher dicht ancinander gedrängter Zellen, die sogenannte pars ciliaris retinae.

Bei den meisten ophthalmoskopischen Choroidalveränderungen spiclen Verïnderungen des Pigmentepithcls eine grosse Rolle. Kast alle I'rocesse nämlich, wolchc im Stroma der Choroidea verlaufen, üben ihre Rückwirkung auf das Choroidalepithel; so z. B. pflegt die bei Morbus Brightii vorkommende Sclerose der Choriocapillaris eine lintfärbung der Pigmentepithelien an der betreffenden Stelle zu veranlassen, die sich auch ophthalmoskopisch durch hellere Flecke z.n erkennen giebt. Ausserden aber entwickeln sich, wie es scheint, selbstständig im Choroidalcpithel eine Reihe von Processen, die meistens in disscminirter Form auftreten, und, indem sie sich über das Niveau des Choroidalepithels erheben, nicht verfehlen können, mit der Stäbchenschicht der Retina in Collision zu gerathen; an der Peripherie des Augenhintergrundes ist dies für das Sehvermögen gleichgültig, geschieht es in der Gegend der macula lutea, so können erhebliche Sehstörungen daraus hervorgehen.

Abgesehen von den von Donders und H. Müller beschriebenen Verdickungen der Glaslamelle gchören hierher eine Reihe von Veränderungen, von denen ich nur einige erwähnen will. In einem Falle z. B. von totaler Netzhautablösung durch einen sarcomatösen Choroidaltumor fand sich am aequator bulbi eine eigenthümliche punctirte Zeichnung der Choroidea. Es erhoben sich dort zahlreiche flache Hügel über das Niveau der Choroide: in deren Querschnitten man zwischen Glaslamelle 
und Choroidalepithel eine helle, undeutlich krümliche Masse vorfand. In ler ganzen Ausdehnung der so veränderten Choroidalparthie zeigten die Epithelien ein äusserst eigenthümliches Verhalten: An der freien Oberfläche derselben fand sich auf dom in ihnen enthaltenen Pigment eine ganz hyaline structurlose glasartige Masse abgelagert, welche häufig in allerhand unregelmässigen kolbigen oder zapfenförmigen Figuren das Niveau der Epithelialschicht übcrragte, und auch beim Betrachten von der Fläche sich durch einen eigenthümlichen Glanz bemerklich machte. Häufiger begegnet man im Choroidalepithel dunklen Flecken, welche dadurch entstehen, dass die Epithelien ein abnorm schwarzes Pigment (manchmal in übergrosser Menge) enthalten, während gleichzeitig meistens die Form der einzelnen Zellen etwas unregelmässig ist und die Zellen selbst in grösserer Quantität vorhanden sind; die benachbarten Choroidalepithelien zeigen bald einen auffallenden Pigmentmangel, bald sind sie ganz unverändert. Diese dunklen Hügel scheinen sich meistens ohne wahrnohmbare Entzündungs-Erscheinungen zu entwickeln, dagegen fand ich in einem von Dr. M o or en wegen anhalt:nder heftiger lentzündungen exstirpirtem Auge das Choroidalepithel in seiner ganzen Ausdehnung unregelınässig, dazu kamen aber noch zahlreiche kleine Verwachsungen zwischen Choroidea und Retina mit bedeutender Atrophie der letzteren an diesen Stellen. Der entzündliche Charakter dieser Affection manifestirte sich ferner durch totale Trübung des Glaskörpers in Folge erheblicher Wucherung seiner zelligen Elemente.

Die eben genannten disseminirten Verwachsungen zwischen Choroidea und Retina mit bedentender secundärer Atrophie der letzteren bilden den Lebergang zu gewissen Formen von Netzhautpigmentirung. Die anatomische Untersuchung hat gezeigt, dass Atrophie und Pigmentirung der Retina häufiger vorkommt, als man 
der ophthalmoskopischen Untersuchung nach erwarten konnte, eben weil die Retina sehr atrophirt sein und viel Pigment enthalten kann, ohne dic für Netzhautpigmentirung characteristischen Zeichen darzubieten, d. h. ohne Pigmentanhäufung an ihren Gefässen. Der allatomische Vorgang hierbei ist der, dass durch chronischentzündliche Processe an der Innenseite der Choroidea theils ausgedehnte Verblebungen zwischen Choroidea und Retina bewirkt, theils eine Durchtränkung und Aufquel. lung der Retina durch Lxsudatflüssigkeiten eingeleitet wird. Diese Durchtränkung führt nun im weiteren Verlaufe $\mathrm{zu}$ einem vollständigen Zugrundegehen aller specifischen Retinalelemente, so dass schliesslich von der Retina weiter nichts übrig bleibt, als ein areolares Netzwerk bindegewebiger Natur. Findet nun gleichzeitig eine Wucherung des Choroidalepithels statt, so können die neu entwickelten Zellen leicht in die lïckenhafte Substanz der atrophischen Netzhaut hineinwachsen und sich wohl auch darin weiterentwickeln. Man findet damn die atrophische Retina reichlich durchsprengt mit rundlichen Zellen, welche ein durch seine dunkle Färbung ausgezeichnetes Pigment enthalten; dieselbe bietet jedoch, bei schwacher Vergrösserung von der Fläche betrachtet, keineswegs die charakteristischen Zeichen dar, an denen wir bei der ophthalmoskopischen Lntersuchung die Pigmentirung der Retina erkennen. Das Bild der pigmentirten Retina entwickelt sich erst, wenn Pigmentanhäufungen an den Retinalgefässen zu Stande kommen.

Die Retinalgefässe können nämlich bei sehr verschiedenen Processen eigenthümliche Veränderungen ihrer Wandungen erleiden, welche zur Verengerung des GefässLumens mit endlicher vollständiger Obliteration führen, und' zur Pigmentablagerung um die Gefässe besonders zu disponiren scheinen. Sehr verschiedene Vorgänge können daher auch schliesslich zu demselben Resultate, 
zur Pigmentirung der Retina fülıren. Hier will ich nur noch anführen, dass ich mich durch die anatomische Untersuchung auf das Bestimmteste überzengt habe, lass eine durchaus selbstständige Entwicklung von absolut schwarzem, lediglich an die Re. tinagefässe gebundenen I'igment vorkonmt. Ich sah dies in einem Falle, wo (neben Iritis, eigenthümlichen Yeränderungen in der Gegend des Ciliarkörpers, Excavation des Selnerven und Atrophie der nervösen letinal-Elemente), sich in den vorderen Partien der Retina, zwischen Aequator und ora serrata feine Pigmentfiguren zeigten, wolche, wic sich bei genauerer Untersuchung ergab, lediglich an den Retinalgefässen sassen. Auch an Querschnitten konnte man sich überzeugen, dass die absolut schwarzen, nicht in Zellen eingeschlossenen Pigmentmassen nur an Retinalgefässen vorkommen. Letztere zeigten in grösserer Ausdehnung, als die Pigmentirung vorhanden war, eine hyaline Verdickung ihrer Wandungen mit Obliteration der feineren Aeste. Das Choroidalepithel verhiclt sich überhaupt und insonderheit auch in dem, dem pigmentirten Bereiche der Retina entsprechenden Bezirke durchaus norinal.

Es liegt also nahe, die ligmententwicklung der Retina hier mit der eigenthümlichen Veränderung der Retinalgefässe in Verbindung zu bringen.

Uebereinstimmende Veränderungen der Retinalgefässe kommen nun, wic erwähnt, bei sehr verschiedenen Processen vor*), und sind unter anderen anch von mir in Fällen gesehen worden, wo in die atrophische Choroidea wuchernde Choroidalepithelien hineingewachsen waren, während an đen obliterirten Retinalgefässen sich

*) Vergl. Dunders, A. f. O. B. IIl 1 pag. 143, Junge, A.t. O. B. V 1 pag. 62, A. Pagenstecher, Würzburger med. Zeitschrift IIl $399-404$. 
Spuren von Pigmententwicklung fanden. Auch für diese F'älle also möchte es annehmbar scheinen, das die Retinalgefässe bekleidende Pigment als dort entstanden zu betrachten.

Aus alle dem möchte ich vor der Hand keinen anderen Schluss ziehen, als den, dass die anatomischen Befunde über das Vorhandensein von Pigment in der Netzhaut nur sehr vorsichtig klinisch zu verwerthen sein dürften. Vor allen lingen betrachte man nicht jeden Fall, in dem sich anatomisch Pigment in der Netzhait nachweisen lässt, als zur ,pigmentirten Netzhaut" gehörig. Mehr und mohr stellt us sich heraus, dass für die zur pigmentirten Net/hant gehörenden functionellen Stiorungen das Vorhandensein von Pigment in der Retina eben nur ein wichtiges Symptom aber nicht die Ursache ist; denn gar nicht selten findet man dieselbe characteristische Gruppe functioneller Störungen ohne jegliche oder mit nur spurweise angedeuteter Netzhantpigmentirung. 